\title{
Parathyroid hormone resistance from severe hypomagnesaemia caused by cisplatin
}

\author{
Adam R. Puchalski, Mary Beth Hodge \\ Division of Endocrinology, Lahey Hospital and Medical Center, Burlington, United States
}

Key words: hypoparathyroidism; parathyroid; magnesium; calcium

We present a case of a 71-year-old male with recently diagnosed, limited-stage, small cell lung cancer status post chemotherapy and radiation, who presented with numbness of his hands, feet, and the area surrounding his mouth. The patient had undergone three cycles of chemotherapy and radiation with cisplatin etoposide, with the last cycle occurring one week prior to admission. In addition to the above symptoms, he also had painful calf and hand contractures and spasms. He denied chest pain, shortness of breath, or palpitations.

Of note, he had been suffering from low calcium and low magnesium since starting chemotherapy and was on home supplements for both. He had no prior history of calcium disorders or any endocrinopathies.

On physical exam, Trousseau sign was positive with use of a blood pressure cuff. He also had hyperreflexia in both upper and lower extremities.

Blood work on admission showed an undetectable $\mathrm{Mg}$ level, a calcium level of $6.0 \mathrm{mg} / \mathrm{dL}$ (corrected $6.6 \mathrm{mg} / \mathrm{dL}$ ), albumin level of $3.2 \mathrm{mg} / \mathrm{dL}$, and an ionised calcium of $0.62 \mathrm{mg} / \mathrm{dL}$. Vitamin D level was normal and serum PTH was elevated at $128 \mathrm{pg} / \mathrm{mL}$.

He was treated with both intravenous magnesium and calcium boluses and admitted into the hospital. His calcium on discharge was $6.7 \mathrm{~g} / \mathrm{dL}$ (no correction given albumin not measured) with a magnesium level of $1.7 \mathrm{mg} / \mathrm{dL}$. He was treated with a total of $10 \mathrm{~g}$ of intravenous magnesium and $6 \mathrm{~g}$ of intravenous calcium during his hospital stay.

The patient was discharged home with oral calcium carbonate and oral magnesium oxide. He was scheduled to follow up in an oncology clinic in one week to have repeat calcium and albumin levels drawn.
Cisplatin is a chemotherapy drug that belongs to the platinum-analogues class. It inhibits DNA synthesis. Cisplatin is known for having many adverse effects including nephrotoxicity, ototoxicity, and numerous electrolyte disturbances, including hypomagnesaemia.

Magnesium plays an important role in parathyroid hormone physiology and calcium homeostasis. Like calcium, it is a divalent cation and binds to the calcium sensing receptor, subsequently activating it $[3,4]$.

Cisplatin causes renal tubular damage and necrosis, leading to increased renal wasting of magnesium. Additionally, it causes damage to calcium and magnesium sensing receptors at the level of the thick ascending loop of Henle and distal convoluted tubules [1]. Therefore, severe hypomagnesaemia that follows leads to both parathyroid hormone resistance and decreased secretion from the parathyroid glands.

The release of PTH from the parathyroid gland and the end-organ effect of PTH is based on magnesium-dependent Cyclic Adenosine Monophosphate (C-AMP) generation which is defective in severe hypomagnesaemia [1]. This leads to decreased calcium reabsorption from the kidneys, decreased parathyroid hormone-mediated calcium release from bones, and decreased vitamin $\mathrm{D}$ production leading to decreased calcium absorption from the gut [2-4].

This can be life threatening. In such patients, calcium and magnesium levels need to be monitored very closely, and the patients should probably be on oral supplements. If this is not possible, then other chemotherapy regimens should be considered for further treatments of such patients. 


\section{References}

1. Njoh RF, Nguyen A, Sadigh M. Cisplatin Induced Hypomagnesemic Hypocalcemia-A Case Report and a Review of the Pathophysiology. J Clin Case Rep. 2013; 3: 259, doi: 10.4172/2165-7920.1000259.

2. Rude RK, Oldham SB, Singer FR. Functional hypoparathyroidism and parathyroid hormone end-organ resistance in human

magnesium deficiency. Clin Endocrinol (Oxf). 1976; 5(3): 209-224, doi: 10.1111/j.1365-2265.1976.tb01947.x, indexed in Pubmed: 182417.

3. by Pa. Parathyroid Gland and Calcium and Phosphate Regulation. In Molina PE. ed. Endocrine Physiology. McGraw-Hill Education 2018: 105.

4. Bilezikian JP. Hypoparathyroidism. J Clin Endocrinol Metab. 2020; 105(6), doi: 10.1210/clinem/dgaa113, indexed in Pubmed: 32322899. 